\title{
Metaphase Plate
}

National Cancer Institute

\section{Source}

National Cancer Institute. Metaphase Plate. NCI Thesaurus. Code C13326.

The plane of the spindle approximately equidistant from the two poles along which the chromosomes are lined up during mitosis or meiosis. 